\title{
Comparative study of acute and perforated appendicitis
}

\author{
Abstract \\ Introduction: The aim of this study was to create a model for the identification of a \\ perforated appendicitis.
}

Patients and Method: All consecutive patients who have undergone an appendectomy in the DY Patil Medical Hospital, Pune between January 1, 2015 and July 31, 2017 were included in a retrospective cohort study. Baseline patient characteristics, history and laboratory data were collected. Variables discriminating perforated from nonperforated appendicitis were identified using univariate and multivariable analyses. These items were used to create a model to predict perforation.

Results: A total of 498patients were included in the study. In the univariate analysis leukocyte count (LC), C-Reactive Protein levels (CRP), Erythrocyte Sedimentation Rate levels (ESR), days of symptoms and temperature were identified as predictors of perforated appendicitis. A predictive model was created using CRP, LC, ESR, duration of abdominal pain, and temperature. The predicted probability $(\mathrm{P})$ of a perforated appendicitis can be calculated from the following model: $(P)=1 /(1+\mathrm{e}(-(-$ $2.788+0.012 * \mathrm{CRP}+0.207 *$ days with complaints)))

Conclusion: Perforation of appendicitis can be predicted from the CRP level and the duration of abdominal pain. These findings might influence the choice between conservative or surgical treatment of appendicitis, and could provide guidance in the early start of antibiotics.

Keywords: abdominal pain, appendicitis, antibiotics, diagnosis, malignancy
Volume 5 Issue I - 2017

\section{Kandarpa Akhil \\ DY Patil Medical Hospital and Research Centre, India}

Correspondence: Kandarpa Akhil, DY Patil Medical Hospital and Research Centre, D-102 Wisdom Park, Pimpri Pune, India, Tel 950-385-154-6,Email akhilmurthy@gmail.com

Received: September 05, 2017 | Published: October 20, 2017

\section{Introduction}

To date, surgery remains the cornerstone in the treatment of appendicitis. However, recent research has revealed that antibiotic treatment might be an appropriate alternative in selected cases. ${ }^{1,2}$ Conservative management with antibiotics causes suppression of bacterial infection by regression of lymphoid hyperplasia. Thereby it might prevent ischemia and bacterial invasion in the early stage of appendicitis. ${ }^{3}$ The complication rates in conservative management increase with perforation leading to higher failure rates in treatment. This especially holds true for children, in whom antibiotics fail in 15 to 25 percent of cases with perforated appendicitis. ${ }^{4}$ Moreover, previous studies have revealed a 3.5 to tenfold increase in mortality rate following perforation..$^{5-7}$ Therefore, accurate pre-operative diagnosis of perforation is important. In patients with suspected appendicitis, inflammatory markers like leukocyte count (LC) and C-Reactive Protein level (CRP) provide as much diagnostic information as clinical findings, such as body temperature and duration of abdominal pain. ${ }^{8}$ In children CRP values aid in the diagnosis of perforated appendicitis. Whether LC may discriminate simple from perforated appendicitis in the pediatric population remains uncertain..$^{9-13}$ Despite previous investigations it remains unclear whether LC, CRP levels and Erythrocyte Sedimentation Rate (ESR) levels combined with body temperature and duration of abdominal pain have a predictive value in diagnosing a perforated appendicitis in unselected patient groups

\section{Aim and objective}

The aim of this study was to determine the predictive value of
LC, CRP levels and ESR levels, combined with body temperature and duration of abdominal pain for the discrimination of simple and perforated appendicitis in patients with acute appendicitis.

\section{Patients and methods}

All consecutive patients who underwent an appendectomy for an acute appendicitis at the D Y Patil Medical Hospital, Pune between January 1, 2015 and July 31, 2017, were included in this retrospective cohort study. All records with the appropriate codes for open and laparoscopic appendectomy were screened for eligibility. The eligibility criteria were a histology report showing appendicitis. Patients were excluded when the removed appendix was not inflamed $(\mathrm{N}=27)$, when a malignancy was present $(\mathrm{N}=4)$ or when there were signs of chronic infection in patients with chronic abdominal pain which were treated with an appendectomy $(\mathrm{N}=5)$. Baseline characteristics including gender, age, and time interval between presentation at the Emergency Department and surgery were collected from patient files. Leukocyte count (LC), C-Reactive Protein levels (CRP), Erythrocyte Sedimentation Rate levels (ESR), temperature at presentation, and the duration of abdominal pain as recorded during inspection at the ED were also collected from the patient records. Information concerning the type of surgery (open versus laparoscopic procedure), condition of the appendix (inflamed, necrotic, infiltrate), and the presence of a perforated appendix was collected from surgical reports. Histology reports were screened for presence of inflammation and perforation. Patients were stratified into 2 groups; non-perforated appendicitis and perforated appendicitis. The perforated appendicitis group consisted of patients whose surgical report noticed a perforated appendix, abscess formation or purulent peritoneal fluid. There is controversy 
in literature on how to define a perforated appendicitis, which might either be based on the surgical report ${ }^{2,4,7,9,14}$ or the histology report. ${ }^{10,13}$ A second rationale for using the surgeon's findings as gold standard is that after-treatment is based upon the surgeon's opinion. Patients with perforated appendicitis received three days of intra-venous antibiotics with Cefazolin and Metronidazole. A comparison of surgeon's findings and histology reports of perforated appendicitis was added.

\section{Data analysis}

Analysis was performed using the Statistical Package for the Social Sciences (SPSS). First, univariate analysis was performed by comparing characteristics between ruptured and simple appendicitis using Mann-Whitney U-test for numeric variables (e.g., age, ESR, CRP, LC, temperature, duration of abdominal pain, surgical delay), and Fisher exact test for categorical variables (e.g., gender). Next, multivariable logistic regression analysis was performed in order to model the relation between different covariates and the occurrence of perforation according to the surgeon. For this, a stepwise regression model with backward elimination (likelihood ratio) of covariates was developed. All items included in the univariate tests were entered in the initial model as covariate. After each step, the covariate with the worst predictive value based upon a log likelihood test was removed until the model with the best fit remained. The classification cut-off for elimination was set at $\mathrm{p}=0.5$.

The best model was selected based upon the criterion

i. Highest Nagelkerke $\mathrm{R}^{2}$,

ii. Best goodness-of-fit according to Hosmer and Lemeshow (HL $\chi^{2}$ and p-value), and iii. Area under the Receiver Operating Curve of the calculated probability $(\mathrm{P})$. The coefficients of the final multivariable model were used to create a formula for calculation of predicted perforation.

\section{Results}

\section{Overall study cohort}

A total number of 498patients who underwent appendectomy with an initial histology report showing appendicitis were included (Table 1). One hundred and five patients had a perforated appendicitis according to the attending surgeon $(21.1 \%)$. The median age was 28.9years (P25-P75 15.9-44.6years). The number of patients under sixteen years of age was $127(25.5 \%)$ and $293(58.8 \%)$ of the patients was male. The appendectomy was performed laparoscopically in 331 patients $(66.5 \%)$. A discrepancy between the surgical report and the histology report was found in 18 percent of cases. In this study the median duration between presentation and surgery was six hours (P25-P75 4-11hours). There was no association between delay and perforation. However, patients with a perforated appendicitis reported a longer period of abdominal pain. $(\mathrm{p}<0.001$; Table 1$)$. Univariate analysis of possible predictors between the groups with perforated appendicitis and non-perforated appendicitis is shown in Table 1. No statistically significant differences were noted between both groups with respect to age, age groups and surgical delay. There is a significant difference between the groups treated by open or laparoscopic appendectomy, with more perforations in the open group. Patients with a perforated appendicitis had higher LC (P 0.011), CRP level $(\mathrm{P}<0.001)$, ESR level $(\mathrm{P}<0.001)$ and temperature $(\mathrm{P}<0.001)$ than patients with a simple appendicitis (Table 1).

Table I Univariate analysis of possible predictors between the groups with perforated appendicitis and non-perforated appendicitis*

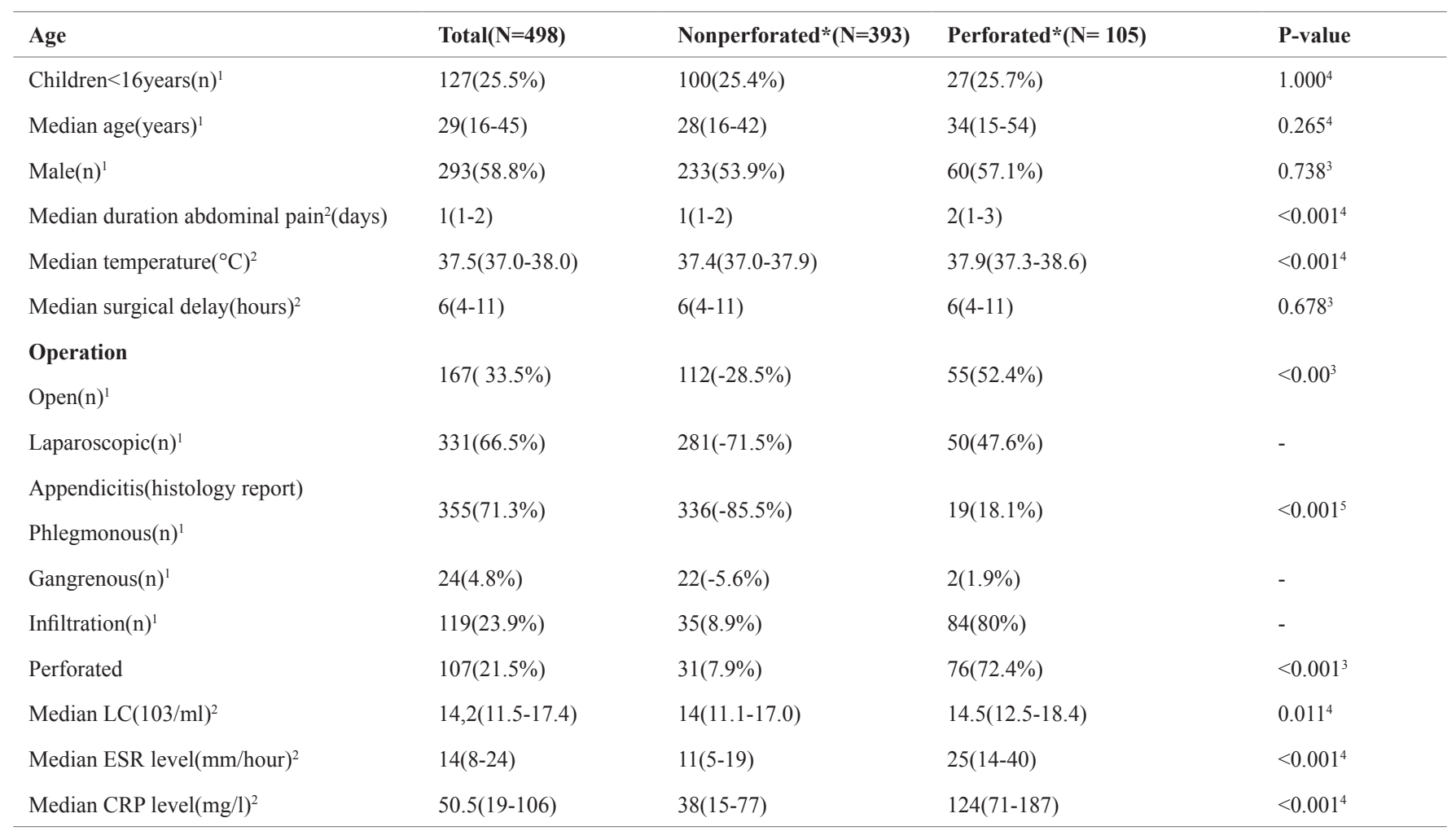


The items age, gender, surgical delay, LC, ESR, CRP, temperature, and days with abdominal pain were entered into the initial logistic regression model. During backward stepwise selection, the following items were eliminated in consecutive order: gender $(\mathrm{p}=0.508)$, temperature $(p=0.450)$, ESR $(p=0.284)$, and surgical delay $(p=0.130)$. The model was further refined by a second selection model using the remaining covariates age, LC, CRP, and duration of abdominal pain. Age $(p=0.505)$ and LC $(p=0.109)$ were subsequently eliminated following the backward selection process. The final model included CRP and duration of abdominal pain and was characterized by a Nagelkerke R-square value of 0.244 and a Hosmer and Lemeshow goodness of fit with a Chi-square value of 9.099 and a P-value of 0.334 . The specificity of this model is $96.3 \%$; negative and positive predictive values are $83.7 \%$ and $66.7 \%$, respectively. The overall accuracy is $82.2 \%$. The regression coefficients for the final regression model are shown in Table 2A. From these coefficients, the predicted probability $(\mathrm{P})$ of a perforated appendix was calculated using the following formula: Figure 1 shows the association between CRP levels and observed perforations in the entire study population. From this Figure it clear that the correlation between CRP and the risk of perforation is not influenced by the leukocyte count (panel A) or the ESR (panel B) (Table 2).

Table 2 Logistic regression model for the prediction of ruptured appendicitis based on surgical reports

\begin{tabular}{llll}
\hline \multirow{2}{*}{ CRP } & P-value & $\begin{array}{l}\text { Regression } \\
\text { coefficient }\end{array}$ & Odds ratio(95\% Ci) \\
\cline { 2 - 4 } & $<0.001$ & 0.012 & $1.012(1.008-1.016)$ \\
\hline $\begin{array}{l}\text { Days with } \\
\text { abdominal pain }\end{array}$ & 0.029 & 0.207 & $1.230(1.021-1.481)$ \\
CRP & $<0.001$ & 0.014 & $1.014(1.009-1.019)$ \\
$\begin{array}{l}\text { Days with } \\
\text { abdominal pain }\end{array}$ & 0.005 & 0.294 & $1.093(1.093-1.646)$ \\
\hline
\end{tabular}

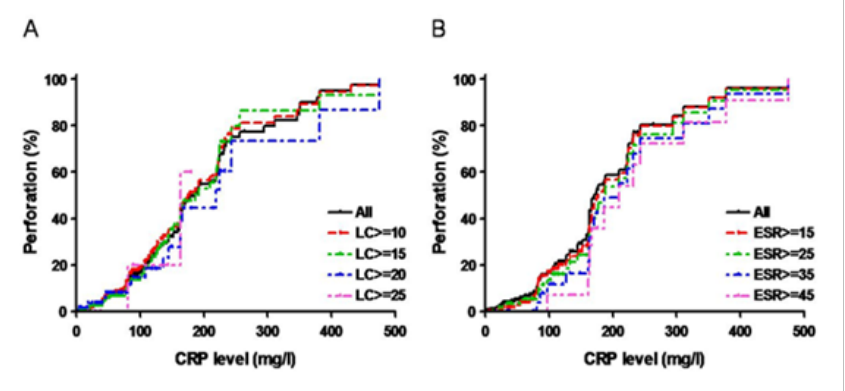

Figure I Perforation rates for increasing CRP levels, different LC levels (panel A) and for different ESR levels (Panel B).

\section{Discussion}

The aim of this study was to create a predictive model for perforated appendicitis using disease history, three laboratory examinations and body temperature. LC, CRP levels, ESR levels, days of symptoms and temperature were statistically significantly higher in patients with a perforated appendicitis. Most studies in the literature focus on predicting appendicitis. ${ }^{8,10,15,16}$ Fewer studies look at the differences between simple and perforated appendicitis. These studies found that LC, granulocyte count and the CRP-level have a discriminatory capacity for perforated appendicitis as compared to simple appendicitis. ${ }^{1,8,10,16}$ In our study, CRP-levels weighted most heavily in the predictive model. The percentage of perforated appendicitis in this study was approximately $21 \%$, which is in concurrence with literature data. $14,15,16$ Visual signs of perforation as observed by the surgeon during surgery were used, to define perforation. Most studies in literature defined their perforated appendicitis group based on surgical reports. Fewer studies defined this group based on the histology report $^{10,13}$ and some combined the surgical report with the histology report. ${ }^{11}$ Based on the literature and the ICD10 we chose to use the surgical report. Furthermore, the after-treatment was based upon this opinion in the surgical report. The discrepancy between the surgical report and the histology report, which was found in $18 \%$ of the cases, could be explained by mechanical damage to the appendix, or by the fact that the surgeon did not record the perforation nor the presence of purulent fluid, or the histology investigation was incomplete because the request was not to search for a perforation but only for appendicitis. Because of this discrepancy, we decided to repeat the data analysis with perforation based on the histology report. This final model included CRP and duration of abdominal pain and was characterized by a Nagelkerke R-square value of 0.336 and a Hosmer and Lemeshow goodness of fit with a Chi-square value of 6.809 and a P-value of 0.557 . The specificity of this model is $85.4 \%$; negative and positive predictive values are $83.1 \%$ and $71.8 \%$, respectively. The overall accuracy is $81.7 \%$. The regression coefficients for this final regression model are shown in Table 2B. From these coefficients, the predicted probability $(\mathrm{P})$ of a perforated appendix was calculated using the following formula: Predicted probability $(\mathrm{P})=1 /(1+\mathrm{e}(-(-$ $4.054+(0.014 * \mathrm{CRP})+(0.294 *$ days of abdominal pain $)))$. Despite the discrepancy, the final results of both analyses are almost similar. The coefficients in the formula are only slightly affected.

We therefore conclude that our model, in which the surgeon's observation of the presence of perforation per-operatively, is valid. In this study we found a statistically significant correlation between perforation and the choice of surgical intervention, e.g. open or laparoscopic $(\mathrm{P}<0.001)$. We cannot rule out a selection bias in which an open approach might be chosen more quickly in patients being severely ill. Furthermore, the Cochrane review, comparing open versus laparoscopic appendectomy, suggest a nearly threefold increase of intra-abdominal abscesses after a laparoscopic appendectomy, which might lead to some Surgeons choosing open appendectomy more often. ${ }^{17,18}$

\section{Conclusion}

In conclusion, increased CRP, LC, ESR and temperature were found to be predictive of perforation in case of appendicitis. Also, a reliable model including CRP level and duration of abdominal pain, could be created, which can predict the probability of perforation in case of appendicitis. Based on such parameters an increased risk of perforated appendicitis can be established, leading to an early start of antibiotic therapy before surgery.

\section{Acknowledgements}

None.

\section{Conflict of interest}

The author declares no conflict of interest. 


\section{References}

1. Hansson J, Korner U, Khorram-Manesh A. Randomized clinical trial of antibiotic therapy versus appendicectomy as primary treatment of acute appendicitis in unselected patients. Br J Surg. 2009;96(5):473-481.

2. Yamini D, Vargas H, Bongard F. Perforated appendicitis: is it truly a surgical urgency? Am Surg. 1998;64(10):970-975.

3. Abes M, Petik B, Kazil S. Nonoperative treatment of acute appendicitis in children. J Pediatr Surg. 2007;42(8):1439-1442.

4. Aprahamian CJ, Barnhart DC, Bledsoe SE, et al. Failure in the nonoperative management of pediatric ruptured appendicitis: predictors and consequences. J Pediatr Surg. 2007;42(6):934-938.

5. Andersson RE. The natural history and traditional management of appendicitis revisited: spontaneous resolution and predominance of prehospital perforations imply that a correct diagnosis is more important than an early diagnosis. World J Surg. 2007;31(1):86-92.

6. Velanovich V, Satava R. Balancing the normal appendectomy rate with the perforated appendicitis rate: implications for quality assurance. Am Surg. 1992;58(4):264-269.

7. Blomqvist PG, Andersson RE, Granath F, et al. Mortality after appendectomy in Sweden, 1987-1996. Ann Surg. 2001;233(4):455-460.

8. Andersson RE, Hugander AP, Ghazi SH, et al. Diagnostic value of disease history, clinical presentation, and inflammatory parameters of appendicitis. World J Surg. 1999;23(2):133-140.

9. Okamoto T, Sano K, Ogasahara K. Receiver-operating characteristic analysis of leukocyte counts and serum C-reactive protein levels in children with advanced appendicitis. Surg Today. 2006;36(6):515-518.
10. Wu HP, Lin CY, Chang CF. Predictive value of C-reactive protein at different cutoff levels in acute appendicitis. Am J Emerg Med. 2005;23(4):449-453.

11. Williams RF, Blakely ML, Fischer PE, et al. Diagnosing ruptured appendicitis preoperatively in pediatric patients. J Am Coll Surg. 2009;208(5):819-825.

12. Rothrock SG, Pagane J. Acute appendicitis in children: emergency department diagnosis and management. Ann Emerg Med. 2000;36(1):3951 .

13. Beltran MA, Almonacid J, Vicencio A. Predictive value of white blood cell count and C-reactive protein in children with appendicitis. J Pediatr Surg. 2007;42(7):1208-1214

14. Ponsky TA, Huang ZJ, Kittle K. Hospital- and patient-level characteristics and the risk of appendiceal rupture and negative appendectomy in children. JAMA. 2004;292(16):1977-1982.

15. Zimmerman MA, Selzman $\mathrm{CH}$, Cothren C. Diagnostic implications of C-reactive protein. Arch Surg. 2003;138(2):220-224.

16. Andersson RE. Meta-analysis of the clinical and laboratory diagnosis of appendicitis. Br J Surg. 2004;91(1):28-37.

17. Oliak D, Yamini D, Udani VM, et al. Can perforated appendicitis Be diagnosed preoperatively based on admission factors? J Gastrointest Surg. 2000;4(5):470-474

18. Sauerland S, Lefering R, Neugebauer EA. Laparoscopic versus open surgery for suspected appendicitis. Cochrane Database Syst Rev. 2004;18(4):CD001546. 